\title{
4. Der manische Patient
}

\author{
Sophie Sommerick
}

Manische Patienten stellen durch ihre auffälligen Verhaltensmuster den Psychiater in einer Klinik vor eine Reihe von Herausforderungen. Symptome einer Manie reichen von deutlich gehobener, heiterer Stimmung, grenzenlosem Optimismus, Antriebssteigerung, Rededrang, sexualisiertem Verhalten, Kaufrausch und Größenideen bis zu einer oft unangenehmeren gereizten Stimmungslage mit ablehnendem, fremdaggressivem, distanzgemindertem Verhalten. Zudem besteht meist eine geminderte oder fehlende Krankheits- und Behandlungseinsicht. Medikamentöse Therapien lehnen die Patienten zu Beginn oft ab und wenn sie bereit sind, Medikamente einzunehmen, tritt der antimanische Effekt erst verzögert ein. Der Aufbau einer therapeutischen Beziehung, die Interaktion mit dem Patienten und die gemeinsame Arbeit des Teams stellen daher gerade zu Beginn die mitunter wichtigsten Bausteine der Therapie dar. Als Arzt in der psychiatrischen Klinik wird man in unterschiedlichen Situationen mit akut manischen Patienten konfrontiert. Zum einen im Erstkontakt in der Notaufnahme: Hierbei steht der Arzt oft den Problemen Fremdaggression, Distanzminderung und Ablehnung gegenüber und soll möglichst Vertrauen aufbauen und zu einer Behandlung motivieren. Und zum anderen als behandelnder Arzt in der Akutphase der ersten Tage bis Wochen auf der Akutstation: Im Vordergrund stehen vor allem die Schaffung einer tragfähigen Arzt-Patient-Beziehung, die Motivation zur Behandlung und das Erarbeiten eines individuellen Behandlungskonzeptes.

Schwerpunkt dieses Kapitels soll nicht die medikamentöse Differenzialtherapie sein (vgl. hierzu Literaturempfehlung). Im Folgenden sollen vielmehr nicht-medikamentöse und interaktionelle Möglichkeiten des Arztes beleuchtet werden v.a. mit dem Ziel, Berufseinsteiger besser auf gelegentlich unangenehme Situationen vorzubereiten und praktisch anwendbare Ratschläge zu geben.

\section{Umgang mit bedrohlich auftretenden Patienten}

Als oberstes Gebot gilt: Selbstschutz geht immer vor! Oft werden manische Patienten durch die Polizei und die Feuerwehr in die Notaufnahme gebracht. Man sollte immer nach Fremdaggression im Vorfeld und während der Fahrt ins Kran- 
kenhaus fragen. Bei beschriebener oder vorbekannter Fremdaggression bzw. auch bei „ungutem Bauchgefühl“, eindeutigen Zeichen von Anspannung, expliziter Ablehnung der stationären Behandlung bei ggf. bestehender Notwendigkeit der Unterbringung nach dem Psych-KG sollten Sie die Polizei immer bitten, so lange zu bleiben, bis die Situation geklärt ist, der Patient auf der Station angekommen ist und man vor Ort entschieden hat, ob weitere Maßnahmen (z.B. 1:1-Betreuung, Fixierung oder Zwangsmaßnahmen) notwendig sind.

Generell empfiehlt es sich bei manischen Patienten, auch wenn sie nicht von der Polizei vorgestellt werden, jemanden mit in das Erstgespräch zu nehmen oder die entsprechenden Notfallalarmiersysteme bei sich zu tragen, wenn die Klinik mit einem Alarmsystem ausgestattet ist.

\section{Kontaktaufbau zum manischen Patienten}

Der erste Patientenkontakt ist oft entscheidend zur Vertrauensförderung für die gesamte Behandlung. Es ist Ziel, den Patienten zu einer freiwilligen Behandlung zu motivieren.

Hierfür ist wichtig, sich zu überlegen, was einem manischen Patienten die Situation der Noteinweisung in die Psychiatrie erleichtern könnte. Bis zum Gespräch mit dem Psychiater sind die Patienten vermutlich auf viel Ablehnung gestoßen und können aufgrund der deutlich eingeschränkten Krankheitseinsicht, der deutlichen Kritikminderung und der Risikobereitschaft die Ablehnung durch die Umwelt kaum verstehen.

- Es ist hilfreich, sich zunächst einzufühlen. Wie reagiert der Patient überhaupt auf den Kontakt mit dem Arzt? Kann er sich auf ein Gespräch einlassen? Welche Themen spricht der Patient von sich aus an, sind ihm also wichtig?

- Schenken Sie dem Patienten Aufmerksamkeit, ertragen Sie ein bisschen seine Logorrhoe und haken Sie ab und zu im Gesprächsfluss nach. Das gibt dem Patienten das Gefühl, dass wirklich Interesse an dem Gesagten besteht.

- Versuchen Sie auch bei deutlichen formal gedanklichen Störungen des Patienten, ein Gespräch zu entwickeln. Die reine Abfrage des psychopathologischen Befundes ist aufgrund von Konzentrationsmangel, Ablehnung und Unruhe oft weder durchführbar noch zielführend. Die wichtigsten Befunde erhält man meist schon durch Beobachten und Zuhören. Natürlich müssen wichtige Dinge wie Suizidalität abgeprüft werden, aber auf Rechenaufgaben zur Konzentrationsprüfung kann beim Erstkontakt meist verzichtet werden.

- Benutzen Sie möglichst einfache Umgangssprache.

- Lassen Sie Lob, Anerkennung und Wertschätzung mit in das Gespräch einfließen. Betonen Sie, dass Ihnen das Wohl des Patienten am Herzen liegt.

- Fragen Sie gezielt nach Wünschen des Patienten in der akuten Situation. Telefonate mit Angehörigen, ein Becher Wasser o.ä. aktiv anbieten.

- Manchmal hilft „Ablenkung“, um eine Situation zu entschärfen und einen vertraulichen Zugang zum Patienten zu erhalten. Die berühmte „Deeskalationszigarette“ sollte ermöglicht oder angeboten werden. Manche Akutstation hat einen angeschlossenen Garten, in welchem man eine vertraulichere Atmosphä- 
re für ein Gespräch schaffen kann. Auch die Bank vor der Notaufnahme ist manchmal entspannter für den Patienten als die räumlichen Gegebenheiten in der Klinik.

- Es gibt auch Situationen, in welchen ein Mitglied des Pflegepersonals einen deutlich besseren Zugang zum Patienten herstellen kann. Man sollte nicht zu stolz sein, diesen Vorteil zu nutzen. Manchmal kennt das Pflegepersonal den Patienten schon, den man als junger Arzt zum ersten Mal trifft, oder „die Chemie stimmt gerade besser“. Beobachten Sie das Gespräch und bitten Sie den entsprechenden Mitarbeiter, wichtige Fragen anzusprechen. Wenn Sie so als „Team“ auftreten, haben Sie möglicherweise auch einen besseren Zugang zum Patienten.

- Sprechen Sie gegenüber dem Patienten an, wenn Sie sich von ihm bedroht fühlen und fragen sie ihn ggf. was er denn selbst beisteuern könne, um Sie zu schützen.

\section{Eigene Ressourcen erkennen}

Gerade zu Berufsbeginn ist es schwierig, seine Rolle als Arzt/Psychiater zu finden und diese im Patientenkontakt gezielt einzusetzen. Sie sollten sich jedoch bewusst machen, wie Sie auf andere wirken. Dadurch können Probleme, aber auch Möglichkeiten in der Interaktion entstehen.

\footnotetext{
Beispiele

Ein großer kräftiger Arzt kann möglicherweise zunächst bedrohlich auf einen Patient wirken. Ressource kann jedoch sein, dass er eher als Autorität wahrgenommen wird und sich im Notfall ggf. besser wehren kann.

Demgegenüber wirkt eine kleine, junge Ärztin oft erst einmal nicht bedrohlich oder provozierend und kann daher möglicherweise zu manchem Patienten schneller einen vertraulichen Kontakt herstellen. Dafür sieht sie sich möglicherweise mit Distanzminderung und sexuellen Anspielungen konfrontiert.
}

Natürlich zählen für solche Aspekte einer Beziehungsaufnahme nicht nur Äußerlichkeiten, es soll jedoch verdeutlichen, dass jeder von uns auf z.T. recht unterschiedliche Weise von anderen wahrgenommen wird. Insgesamt hilft es am besten, sich nicht zu verstellen, sondern seine Ressourcen zu kennen und zu stärken.

\section{Gestaltung des Behandlungsverlaufes}

Ziel ist es, ein gemeinsames individuelles Behandlungskonzept zu finden und zur ambulanten Weiterbehandlung zu motivieren.

- Gehen Sie im medizinisch vertretbaren Rahmen auf Wünsche des Patienten ein. Nehmen Sie Bedenken und Vorurteile gegenüber einer Medikation ernst. Die medikamentöse Therapie entspricht häufig keinem „Raster“. Einnahmezeiten richten sich gelegentlich nach speziellen Wünschen der Patienten oder sind davon abhängig, wann der Mitarbeiter mit dem größten Vertrauensverhältnis zum Patienten Dienst hat. 
- Es ist natürlich bei allen Patienten wichtig, deren Biographie gut zu kennen. Gerade jedoch bei manischen Patienten sind durch die Erkrankung z.T. tragische Veränderungen im Leben aufgetreten. Oft stehen die Patienten vor einem schwierigen Neubeginn, haben Kontakt zu und Anerkennung durch Vertrauenspersonen verloren oder sind enttäuscht vom eigenen Lebenslauf. Trotz Größenideen und Optimismus entwickelt sich im Verlauf meist ein Verständnis für die missgünstige Lage. Betonen Sie Ihre Wertschätzung des Patienten, helfen Sie bei der Suche nach neuen Perspektiven und vor allem: Nehmen Sie eine ggf. unterliegende deprimierte oder verzweifelte Stimmungslage wahr und sprechen Sie diese vorsichtig an.

\section{Wahren Sie soweit wie möglich die Autonomie des Patienten}

- Der manische Patient kostet Zeit! Ständig neue Ideen, Rededrang, wechselnde Affekte, Konflikte mit Mitpatienten oder Verstoß gegen Stationsregeln führen zu einem hohen Gesprächsbedarf. Es empfiehlt sich, Kurzkontakte selbst anzubieten, anfangs auch mehrfach täglich und am besten den nächsten Kontakt (Visite, erneuter Kurzkontakt o.ä.) zeitlich festzulegen.

- Es ist hilfreich, wichtige Gespräche und Behandlungsabsprachen gemeinsam mit Psychologen, Sozialarbeitern oder Mitarbeitern der Pflege durchzuführen. Zudem ist dies eine gute Möglichkeit, sich Feedback von Erfahrenen einzuholen.

- Angehörige/Vertrauenspersonen sollten möglichst von Beginn miteinbezogen werden. Das kann das Vertrauen zum Patienten fördern. Außerdem können dadurch z.B. Ausgänge in Begleitung schneller ermöglicht werden.

- Psychoedukative Gespräche sind gerade zu Beginn oft nicht im Gruppenprogramm der Stationen möglich, sondern müssen in Einzelkontakten geführt werden. Gehen Sie am Anfang auf Symptome ein, die den Patienten möglicherweise selbst stören, wie Schlafstörungen, Unruhe oder Konzentrationsmangel. Im Verlauf sollten die Themenschwerpunkte zunehmend auf Wahnkorrektur, Krankheitsprognose und Notwendigkeit der ambulanten Weiterbehandlung liegen.

- Im Umgang mit Distanzminderung und sexuellen Anspielungen hat jeder sein eigenes Maß, wann eine klare Grenze gesetzt werden muss. Bis zu einem gewissen Grad, kann „Geflirte“ seitens des Patienten genutzt werden, um zum Beispiel zur Medikamenteneinnahme zu bewegen. Wenn es jedoch störend ist, hilft es, betont sachlich zu bleiben. Sollte das nicht ausreichen, sollten Sie das Problem klar ansprechen und den Patienten wieder zurück auf die Behandlung orientieren (z.B. „das geht zu weit“ oder „es geht in diesem Gespräch um die Therapie Ihrer Erkrankung“). Manchmal kann aber auch ein Wechsel des Behandlers nötig werden. Gerade für solche Situationen gilt: Tauschen Sie sich mit Ihrem Team aus und zögern Sie nicht, auch Ihren Oberarzt zu bitten, Sie beim Setzen von Grenzen zu unterstützen. 


\section{Zusammenfassung}

Ihre größte Ressource im Umgang mit manischen Patienten ist oft Ihr Team, nutzen Sie diesen Vorteil. Geben Sie manischen Patienten das Gefühl von Wertschätzung und lernen Sie eigene Ressourcen im Umgang mit diesen Patienten kennen und stärken. Wir sind in diesem Kapitel auf besonders schwierige oder unangenehme Situationen eingegangen wie sie in der Akutbehandlung häufig auftreten. Es sollte aber nicht vergessen werden: Die Behandlung manischer Patienten kann aufgrund des überspringenden Optimismus oft erfrischend sein und den Alltag deutlich aufhellen.

\section{Literaturempfehlung}

Benkert 0, Hippius H (2014) Kompendium der Psychiatrischen Pharmakotherapie. 10. Aufl. Springer Verlag Heidelberg

Berger M (2014) Psychiatrische Erkrankungen. 5. Aufl. Urban \& Fischer München

Dörner K (2013) Irren ist menschlich. 22. Aufl. Psychiatrie Verlag Köln

Ebert D (2011) Psychiatrie systematisch. 8. Aufl. Uni-Med-Verlag Bremen

Jacob G, Lieb K, Berger M (2009) Schwierige Gesprächssituationen in Psychiatrie und Psychotherapie. Urban \& Fischer München 